\title{
Postural Control at Hospital Discharge after Spine Surgery-The Influence of the Type of Surgery, Pain Level, Radiculopathy and Stance Parameters
}

\section{Relvas $\mathrm{H}^{1 *}$, Diniz $\mathrm{A}^{2}$, Fernandes $\mathbf{0}^{3}$ and Oliveira $\mathrm{R}^{4}$}

${ }^{1}$ Portuguese Red Cross School of Health, Lisbon, Portugal

${ }^{2}$ CIPER, Human Kinetics Faculty, Lisbon University, Portugal

${ }^{3}$ Science \& Technology School, Évora University, Portugal

${ }^{4}$ CIPER, Neuromuscular Research Lab., Human Kinetics Faculty, Lisbon University, Portugal

\section{Research Article}

Volume 4 Issue 1

Received Date: July 30, 2020

Published Date: September 02, 2020

DOI: $10.23880 /$ jobd-16000197

*Corresponding author: Henrique Relvas, Portuguese Red Cross School of Health, Lisbon, Portugal, Av. Ceuta 1 Ed. Urbiceuta 1300-125 Lisboa, Portugal, Email: hrelvas@esscvp.eu

\section{Abstract}

Information on postural control has gained recognition as an objective outcome measure after spine surgery. Insights on the patterns of posture and movement exhibited by patients using nonlinear tools can provide useful information on the structure of sway variability, adding value to the traditional quantification of its magnitude. The results should guide efficient therapeutic strategies to promote early recovery. To date, no clinical trial used this protocol at the time of hospital discharge. This study aims to analyse postural control under the acute effect of spine surgery.

Thirty-seven patients submitted to decompressive or fusion spine surgery to treat degenerative pathologies or correct severe deformities were recruited (21 male, 16 female; mean age: 53,4 $\pm 18,1 \mathrm{y}$, range 14-80). Thirty-seven matching healthy volunteers included the control group. All participants performed a standard stabilometric test on a force platform for 180 seconds, patients being tested at the hospital on discharge day. Six more trials were randomly performed in different stance conditions: 1) eyes closed; 2) feet together; 3) performing a mental task; 4) on a high-density foam; 5) on a low-density foam; 6) holding a box. Values of sway range, mean velocity, elliptic area, and sample entropy, were extracted from centre-of-pressure displacement time-series and analysed using parametric statistical methods. Comparisons were made between control values and the effects of surgery, type of surgical procedure, level of back or leg pain, and residual radiculopathy.

During the baseline task (eyes open) patients increased their total area of oscillation $(p=0,016)$, mainly its range in the anteroposterior (AP) direction $(\mathrm{p}=0,010)$; the structure of that variability has less complexity and more regularity compared to control participants, showing lower sample entropy values ( $\mathrm{p}=0,031$ / AP direction). Differences were not significant in the mediolateral (ML) direction, and the mean velocity did not discriminate participants. Eyes closed condition, being on a highdensity foam, or holding a box, exhibited the most robust cumulative significance. Interactions between all the parameters (the type of surgery, level of pain, presence of radiculopathy) may reveal more significant determinants to the postural patterns adopted. However, present data should be interpreted cautiously, being necessary broader samples for each sub-group in future studies, the replication of these measures in later follow-ups, and consider the effects of rehabilitation programs tailored in concordance to the main findings. 


\section{Journal of Orthopedics \& Bone Disorders}

At hospital discharge, patients having surgery for spine conditions show a significant increase in the magnitude of body sway and a postural pattern markedly regular with low complexity. Stabilometric trials performed with eyes closed, on high-density foam, or holding a box, demonstrate superior ability to discriminate patients from controls and should be used preferentially in future trials. Further studies using nonlinear measures after spine surgery are needed to clarify the underlying motor behaviour. Early intervention is recommended to correct postural impairments and facilitate optimal movement variability.

Keywords: Spine Surgery; Quantitative Outcomes; Motor Behavior; Sway Variability; Non-Linear Dynamics

\section{Introduction}

The pain and disability associated with spinal disorders represent a considerable personal and social burden due to their persistent and progressive nature. From the latest studies available, low back pain is the single leading cause of years lived with disability for all ages and both sexes combined, across 126 of the 195 countries under scrutiny [1]. A worsening global trend is also clear, with an increase of about $50 \%$ since the first data collection in 1990 .

Although recommendations from best practice guidelines exist, in line with the evidence for the costeffectiveness of strategies proposed to prevent and treat spinal disorders, a deep contrast occurs in the real practice, whatever the income setting [2].

In particular, surgery for back pain remains a controversial issue, regarding the uncertainty on relative profits and risk of adverse events [3], representing a lack of consistency between the ability to solve back problems or being an additional contributing factor to further spinal dysfunction. The higher amount of costs and health care resources involved adds some arguments to increase the debate.

A broader agreement is achieved though, whenever surgery is proposed in the presence of a severe or progressive neurological impairment [4], or lack of positive outcomes from conservative treatment, with a strong association of symptoms with structural imaging findings, like herniated discs or spinal stenosis [5].

Good practice advocates that indications for spine surgery would be supported by unequivocal criteria, as decisions about the best approach should be driven by updated and high-quality clinical practice guidelines, built on substantial evidence and based on a shared process between clinicians and patients to achieve the best choice [6,7]. The systematic use of a comprehensive and standardised set of outcome measures after spine surgery would help to minimise the gap between evidence and practice, looking to optimise decision-making, especially in the absence of a clear indication for surgery [8].

Although a primary core set of outcomes is consensually based on patient-reported subjective measures like pain intensity, physical functioning, and health-related quality of life [9], reliable and objective measures with clinical significance should also be assessed [10]. Evaluating the impact of surgery on the patterns of posture and movement using kinetic and kinematic measuring tools [11] should provide a closer insight into the biomechanical and neuromuscular functions under the effect of constraints due to surgery.

Analysis of the correlations between subjective outcome scores from the primary core set and the measures of motor behavior parameters would enhance the reliability and usefulness of this information to compare during recovery and guide rehabilitation approaches. Balance and postural control are essential features for performing a multitude of motor tasks while ensuring spinal stability, protecting against injury and pain, and reducing metabolic costs [12,13]. Low back pain has already proven to have a deleterious effect on motor strategies, emphasising higher levels of trunk muscle co-activation, resulting in a rigid postural control as a protective coping scheme, at the expenses of lesser motor behaviour variability, additional overload, and energy waste $[14,15]$. Evidence also points to profound structural alterations of the deep trunk muscles with significant changes in neuromuscular recruitment patterns, including inhibition, delayed onset, atrophy, fatty infiltration and muscle fibre type mutation [16-18].

Patients submitted to spine surgery for back pain may exhibit all these features, expanded by a higher level of pain interference [19] and some form of sensorimotor impairment that may add further compromise to their motor performance [20-23]. Proprioceptive impairment seems to be the main feature, due to some level of radiculopathy remaining from previous neural tissue compression or consecutive to surgical iatrogenicity by afferent denervation and dysfunctional central processing related to the incision aggression, changes in spinal curvatures, or insertion of rigid segments by fusion/arthrodesis of intervertebral segments 


\section{Journal of Orthopedics \& Bone Disorders}

[24].

Posturography is widely used to quantify body swing during upright stance to infer the integration and interaction of various sensorimotor control systems, guiding researchers and clinicians to understand and manage movement and functioning in both healthy and pathological populations. Static posturography, or stabilometry, uses force platforms to collect information about the centre of pressure displacement (CoP - the point of application of the vertical ground reaction force vector, exerted as a reply to foot contact on the platform), measured while maintaining a quiet upright position, during the movement of the body like a single inverted pendulum rotating around the ankle joints [25]. This procedure creates two time-series data sets, each for the CoP trajectory around the anteroposterior (AP) or mediolateral (ML) axis, representing the two-dimensional variability of body sway under the conditions imposed by the experimental setup [26].

Different metrics are proposed to quantify the recorded amount of sway. Classical linear tools using time-domain parameters (e.g., range, area, travelled distance, and velocity of the CoP trajectory) suggest an index of postural stability assuming that less sway reveals an enhanced control $[27,28]$. Nevertheless, the magnitude of this variability around the average fails to account for the structured temporal organisation of motor behaviour [29-31]. Nonlinear dynamics proposes to quantify motor output regularity over a time-series (e.g., sample entropy) as a measure of complexity, being the best representation of its variability [32-34].

Since the first study in 1991 [35] and despite its proven utility to evaluate changes in motor performance due to spinal disorders or the outcomes of its management strategies [36-39], few stabilometric studies have been carried out to date in the scope of spine surgery and only one systematic review [40]. The majority of studies point to increased or faster postural sway as the most common features, when comparing low back patients with healthy subjects. However, the inconsistency is considerable and seems to be dependent on the stance conditions imposed by each experimental protocol.

Guidelines on the conditions under which quiet stance trials should be made on the platform or the parameters for analysing the data have been the issue of profound debate [41]. However, a weak consensus among researchers still precludes effective standardisation. Overall recommendations indicate the eyes-closed condition as more sensitive for identifying alterations in balance behaviour [40], but other possibilities of a mechanic, sensory, or cognitive manipulation raise the interest in exploring diverse standing conditions.
Additionally, previous studies identified a further increase in body sway immediately after fusion surgery that gradually diminishes over time [40]; or a reduction straightway in the case of decompressive procedures, comparing to the pre-operative values $[22,40,42]$. Although an overall improvement in postural control is achieved, a significant difference tends to subsist comparing surgery patients to healthy subjects after 1 to 3 weeks $[43,44] ; 1$ to 3 months $[20,45,46]$; and even in the long-term follow-up of 6 to 12 months [47-49]. In the cases of adolescent scoliosis, it may take 1 to more than 3 years to reach recovery [24,50-52]. Bouche and colleagues [53] found ongoing postural deficits in patients submitted in the long-term to decompressive lumbar surgery, as far as 42 to 83 months.

It appears that the immediate stage after surgery is the one in which the issues that may influence the longterm evolution of patients are emphasised, and particularly residual pain and radiculopathy, and maybe the appropriate moment to collect these data to highlight their effects.

The eyes closed condition was suggested as the most capable of detaching the main features of postural patterns emerging in the early postoperative period. At that time, it seems relevant to assess how patients evolve after surgery and solve their previous impairments, like their dependence on visual clues to compensate for any proprioceptive deficits. Meanwhile, many other parameters need to be considered that may compromise postural control and the future outcome of the surgery, to overcome the remaining discrepancy between patients and healthy subjects in the long-term, as mentioned before. Sipko and colleagues [22] suggest that this transient period is critical to implement therapeutic strategies targeted at facilitating and reinforcing the acquisition of correct motor patterns.

Such a proposition needs to be sustained on a comprehensive evaluation of outcomes using the most appropriate assessment tools. Only two stabilometric studies evaluated the acute effects of spine surgery on postural control, measured within the hospital setting at the time of discharge, both aiming at decompressive surgery alone $[22,42]$. To our knowledge, none of the reviewed studies used nonlinear tools to appraise postural control variability immediately after spine surgery. Linking both approaches could provide a comprehensive understanding of the underlying motor strategies adopted in response to pain and other influences from pathology or surgery [33].

Therefore, this exploratory clinical trial aimed to describe the postural control features of patients who just underwent spine surgery, comparing the results with a control group. Linear (range, area, and velocity) and nonlinear (sample entropy) measures of the CoP displacement were used to 


\section{Journal of Orthopedics \& Bone Disorders}

analyse the influence of the type of spine surgery, the level of reported pain, the presence of signs or symptoms of radiculopathy, and the effect of stance parameters.

It was hypothesised that fusion procedures would negatively influence postural sway in the proportion of its extension, more than decompressive surgery, associated with higher levels of pain and residual radicular impairment. It was consequently expected an increased oscillation with a less complex and more regular pattern of variability, comparing to control values. These changes should be progressively highlighted with increased difficulty imposed by the stance condition, starting from the baseline task (eyes open, feet apart, on a firm surface, performing a single task).

\section{Methodology}

\section{Participants}

Users of the Portuguese Red Cross Hospital (PRCH) were recruited for convenience, sequentially selected from the list of hospital admissions to undergo spine surgery.

Inclusion criteria for the surgery group were subjects of both genders and any age proposed for 1) decompression surgery, involving one or more lumbar intervertebral levels with a combination of discectomy, laminectomy and foraminotomy procedures; 2) fixation surgery -instrumented posterolateral fusion alone or combined with interbody fusion (arthrodesis) after decompression surgery, between one or more lumbar or thoracolumbar intervertebral levels. Surgery was proposed to treat degenerative pathologies or correct severe deformities, such as adolescent idiopathic or adult degenerative scoliosis.

As exclusion criteria, cases of cervical surgery, minimally invasive techniques, or procedures with devices such as interspinous spacers or disc arthroplasty were rejected. Subjects were not included if they had sequelae from a previous history of severe traumatic injury, surgery of the lower limbs, central neurological injury, or systemic pathology associated with balance impairment.

Subjects recruited for convenience among PRCH's employees and users set up the control group, so to match the subjects of the surgery group in terms of gender and age. The exclusion criteria were a traumatic or surgical history involving the spine or lower limbs, systemic pathology with central neurological or balance impairment, and reference to back or neck pain within the last three months.

The PRCH Ethics Committee approved our study, and the participants gave their free and informed written consent to participate in the clinical trial.

\section{Material and Methods}

Participants in both groups performed a standard stabilometric test. Those in the surgery group were tested on the day of hospital discharge. At that time, they have demonstrated criteria that justify the cessation of hospital care: a stabilised clinical condition and functional autonomy to perform basic activities of daily living, such as transfers, sitting, walking, and climbing stairs. Levels of reported back or leg pain through the last hour were recorded. The 0-10 point Numerical Pain Rating Scale (NRS) $[54,55]$ was used to assess pain intensity. Participants were rated according to the presence of any complaint of referred leg pain, motor impairment, or disrupted sensation affecting the lower limbs due to lumbosacral nerve root compromise [56].

During an initial 180-second trial (baseline task), participants were required to maintain a relaxed and natural upright stance on a force platform while they were barefoot with their feet hip-width apart, their arms comfortably extended to the side, and their eyes open. They kept looking at a $5 \mathrm{~cm}$ circular mark on a wall 3 meters ahead at eye level, in a quiet, bright room in the Physiotherapy Department of the hospital. Participants were then asked to randomly perform six more trials, in different conditions while standing on the platform: 1) eyes closed; 2) feet close together; 3) performing a mental task; 4) on a high-density foam; 5) on a low-density foam; 6) holding a small box. A minimum rest period of sixty seconds was applied between trials to avoid fatigue or additional discomfort, during which participants were allowed to lay down, sit, stand, or walk around, as preferable.

The AP and ML directions of the CoP oscillations were acquired using a Bertec ${ }^{\oplus}$ mod. 4060-NC (Bertec Co., Columbus, USA) six-component strain-gauge force platform with a sampling rate of $1 \mathrm{~K} \mathrm{~Hz}$. Data recording started after the initial 5-seconds period to stabilise the position. Each of the 180 -seconds raw signals was then down sampled to 20 $\mathrm{Hz}$, without filtering or any additional processing.

Linear measures of variability used to analyze the CoP displacement time-series included temporal (range [AP/ ML]-the amplitude between the maximum and minimum values of displacement, in each direction; velocity [AP/ $\mathrm{ML}$ ] - the displacement by the test time, in each direction) and spatiotemporal (area - the surface of an ellipse that covers $95 \%$ of the total displacement) parameters [28]; sample entropy (AP/ML) was the nonlinear measure used as the extent of complexity and regularity of the body sway, reflecting the dynamic structure of its variability [57].

Therefore, seven quantitative measures were selected as dependent variables. As mentioned, the values of these variables were recorded under seven task conditions. 


\section{Journal of Orthopedics \& Bone Disorders}

Moreover, the clusters/groups specified for CoP displacement comparison incorporated group (control group and surgery group), type of surgery (decompression surgery and fusion surgery), level of pain (pain lower than 5 and pain 5 or higher in the 0-10 point Numerical Pain Rating Scale) and presence of radiculopathy (without radiculopathy and with radiculopathy).

Statistical analysis was used to describe participant groups and to compare the effects of different task conditions or surgical and clinical parameters on CoP displacement variability.

\section{Statistical Procedures}

The first step consisted of computing descriptive statistics (e.g., frequencies, means, and standard deviations) for the several types of variables and groups included in the study. Next, Shapiro-Wilk's, Levene's, and Mauchly's tests were conducted to examine the normality, the homogeneity of variances, and the sphericity conditions, respectively, of the dependent variables across groups and conditions.

Subsequently, and selecting the baseline task (eyes open), Independent-Samples t-tests, and One-Way ANOVAs (without or with Welch's correction) with Tukey's post-hoc comparisons were performed to evaluate the difference between the dependent variables mean values across groups.

Ultimately, Two-Way ANOVAs for repeated measures and several groups (without or with Greenhouse-Geisser's correction) with Bonferroni's post-hoc comparisons were used to evaluate the difference between the dependent variables mean values for the different conditions and groups. The probability $\mathrm{p}<0,05$ was set as the criterion for statistical significance. This part of the study was undertaken using the IBM SPSS software (version 25, IBM Inc., USA).

\section{Results}

Thirty-seven patients were selected for the surgery group, $21(56,8 \%)$ of whom were male, $16(43,2 \%)$ were female. The ages were between 14 and 80 years old; the average age was $53,4 \pm 18,1$ years. Half of the patients were older than 58 years.

A match was obtained in terms of gender and age range between the participants in both groups: 37 participants $(21$ male, 16 female) were included in the control group (average age 53,4 $\pm 17,8$ years). A considerable similarity in the mean values of age, height, weight, and Body Mass Index was also attained (Table 1).

\begin{tabular}{|c|c|c|c|c|c|c|c|c|c|c|c|}
\hline \multirow{2}{*}{ Age range } & \multicolumn{3}{|c|}{ CG/SG (same values) } & \multicolumn{2}{|c|}{ Age (y) * } & \multicolumn{2}{|c|}{ Height (m) * } & \multicolumn{2}{|c|}{ Weight (kg) * } & \multicolumn{2}{|c|}{ BMI $(\mathrm{kg} / \mathrm{m} 2)$} \\
\hline & n (\%) & q & $\hat{0}$ & CG & SG & CG & SG & CG & SG & CG & SG \\
\hline$<35$ & $5(13,51 \%)$ & 1 & 4 & 20,6 & 19,4 & 1,72 & 1,79 & 62,2 & 69,4 & 21,0 & 21,3 \\
\hline $35-44$ & $6(16,22 \%)$ & 3 & 3 & 38,5 & 38,7 & 1,74 & 1,71 & 74,7 & 76,2 & 24,5 & 25,7 \\
\hline $45-54$ & $4(10,81 \%)$ & 2 & 2 & 49,0 & 49,3 & 1,72 & 1,72 & 72,5 & 72,9 & 24,8 & 24,6 \\
\hline $55-64$ & $12(32,43 \%)$ & 5 & 7 & 60,4 & 60,6 & 1,67 & 1,68 & 72,2 & 82,1 & 25,8 & 29,1 \\
\hline$>65$ & $10(27,03 \%)$ & 5 & 5 & 72,2 & 72,4 & 1,65 & 1,62 & 77,2 & 73,5 & 27,9 & 28,0 \\
\hline Total & $37(100 \%)$ & 16 & 21 & 53,4 & 53,4 & 1,69 & 1,69 & 72,6 & 76,1 & 25,4 & 26,7 \\
\hline & & & & $\pm 17,8$ & $\pm 18,1$ & $\pm 0,11$ & $\pm 0,11$ & $\pm 15,5$ & $\pm 15,3$ & $\pm 4,1$ & $\pm 4,7$ \\
\hline & & & & & & & mean & td value & & & \\
\hline
\end{tabular}

Table 1: Distribution by age range and gender within the control (CG) and surgery (SG) groups (same values for both groups). Mean values of age, height, weight, and Body Mass Index (BMI).

\begin{tabular}{|c|c|c|c|c|c|c|c|}
\hline $\begin{array}{c}\text { Type of spine } \\
\text { surgery }\end{array}$ & n (\%) & o & 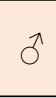 & Age $(y)^{*}$ & Height (m)* & $\begin{array}{c}\text { Weight } \\
\text { (kg)* }\end{array}$ & BMI $(\mathrm{kg} / \mathrm{m} 2)^{*}$ \\
\hline Decompression & $17(45,95 \%)$ & 6 & 11 & $53,1 \pm 18,4$ & $1,73 \pm 0,13$ & $77,9 \pm 14,1$ & $26,0 \pm 3,8$ \\
\hline Fusion (arthrodesis) & $20(54,05 \%)$ & 10 & 10 & $53,7 \pm 18,3$ & $1,65 \pm 0,09$ & $74,6 \pm 16,5$ & $27,3 \pm 5,5$ \\
\hline Total & $37(100 \%)$ & 16 & 21 & $53,4 \pm 18,1$ & $1,69 \pm 0,11$ & $76,1 \pm 15,3$ & $26,7 \pm 4,7$ \\
\hline & & & & \multicolumn{4}{|c|}{${ }^{*}$ mean \pm std values } \\
\hline
\end{tabular}

Table 2: Demographic and anthropometric description of the surgery group. 
There were no major differences in the demographic and anthropometric characteristics between decompression and fusion participants in the surgical group, although fewer women required a decompressive procedure alone. This subgroup had, therefore, a slight tendency to higher and heavier participants (Table 2).

In this sample, surgeries in participants under 50 years old were mainly decompressive at the $\mathrm{L}_{5}-\mathrm{S}_{1}$ level, or corrective, as a treatment of scoliosis. Intervention at the $\mathrm{L}_{4}$ $\mathrm{L}_{5}$ segment was the most frequent, alone, or in combination with other levels (Table 3).

The participants who underwent fusion (arthrodesis) stayed in the hospital twice as long and complained more about back or leg pain, on average.

Half of the participants had signs and symptoms of a lumbosacral radicular syndrome after spine surgery, but not related to any other parameter in this group. Only seven (19\%) of the 37 participants in the surgery group referred residual leg pain at the time of hospital discharge after spine surgery; four of those had no other radicular signs or symptoms. Twelve participants had some degree of motor impairment in the critical muscles of a specific radicular myotome. Nineteen participants were free of any complaint of lumbosacral radicular involvement.

\begin{tabular}{|c|c|c|c|c|c|c|c|}
\hline $\begin{array}{c}\text { Type and levels of spine } \\
\text { surgery }\end{array}$ & n (\%) & q & $\hat{0}$ & $\begin{array}{c}\text { age } \\
\text { (years)* }\end{array}$ & $\begin{array}{c}\text { length of } \\
\text { hospital } \\
\text { stay (days)* }\end{array}$ & $\begin{array}{c}\text { back/ } \\
\text { leg pain } \\
\text { intensity (0- } \\
10 \mathrm{NRS})^{*}\end{array}$ & $\begin{array}{c}\text { lumbosacral } \\
\text { radic. syndr. } \\
(n / \%)\end{array}$ \\
\hline \multicolumn{8}{|l|}{ Decompression } \\
\hline L3-L4 & $1(2,70 \%)$ & 0 & 1 & 72,0 & 2,0 & 4,0 & 1 \\
\hline L4-L5 & $7(18,92 \%)$ & 3 & 4 & 63,3 & 2,0 & 5,1 & 3 \\
\hline \multirow[t]{2}{*}{ L5-S1 } & $9(24,32 \%)$ & 3 & 6 & 43,1 & 1,3 & 3,3 & 5 \\
\hline & $17(45,95 \%)$ & 6 & 11 & $53,1 \pm 18,4$ & $1,7 \pm 0,9$ & $4,1 \pm 2,5$ & $9(53 \% / 17)$ \\
\hline \multicolumn{8}{|l|}{ Fusion (arthrodesis) } \\
\hline lumbar / L4-L5 (x1) & $7(18,92 \%)$ & 2 & 5 & 64,4 & 3,1 & 4,6 & 4 \\
\hline lumbosacral / L5-S1 (x1) & $3(8,11 \%)$ & 1 & 2 & 47,7 & 2,0 & 5,7 & 1 \\
\hline lumbosacral / L4-S1 (x2) & $3(8,11 \%)$ & 3 & 0 & 63,3 & 3,0 & 8,3 & 3 \\
\hline lumbosacral / L3-S1 (x3) & $3(8,11 \%)$ & 2 & 1 & 64,3 & 4,0 & 4,3 & 1 \\
\hline thoracolumbar / T11-L3 (x4) & $1(2,70 \%)$ & 1 & 0 & 50,0 & 4,0 & 7,0 & 0 \\
\hline \multirow[t]{2}{*}{ thoracolumbar / T4-L3 (x11) } & $3(8,11 \%)$ & 1 & 2 & 15,7 & 5,0 & 8,0 & 0 \\
\hline & $20(54,05 \%)$ & 10 & 10 & $53,7 \pm 18,3$ & $3,4 \pm 1,1$ & $5,9 \pm 2,1$ & $9(45 \% / 20)$ \\
\hline Total & $37(100 \%)$ & 16 & 21 & $53,4 \pm 18,1$ & $2,6 \pm 1,4$ & $5,1 \pm 2,4$ & $18(49 \% / 37)$ \\
\hline & & & & & ${ }^{*}$ mean \pm std va & des & \\
\hline
\end{tabular}

Table 3: Distribution and gender by type and levels of procedure within the surgery group. For each item: - mean values of age, hospital stay, and referred back or leg pain; - participants with signs and symptoms of a lumbosacral radicular syndrome (number/percentage per sub-group).

Figure 1 identifies successive clusters of reported back or leg pain in ascending order of intensity from all the surgery participants, each one representing the type and extension of the surgical procedure (single or multiple segments involved). Generally, the distribution followed the progressive impact of the surgery extension and the use of instrumentation for the fusion procedure. Nevertheless, the higher pain reports came from a one-level decompressive case (the oldest participant) and from the group of three women who underwent two-segment fusion $\left(\mathrm{L}_{4}-\mathrm{S}_{1}\right.$ levels $)$, with the highest mean value (8,3/10 NRS). On average, women complained slightly more $(5,8 / 10$ NRS) than men $(4,5 / 10 \mathrm{NRS})$. 


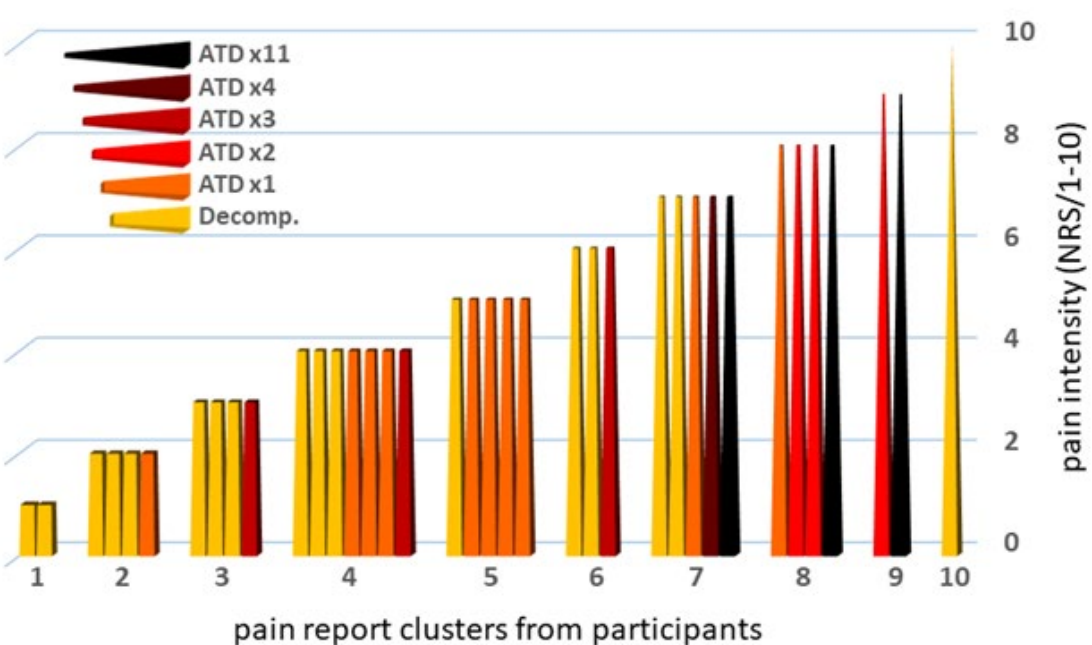

Figure 1: Back or leg pain reports from the 37 patients of the surgery group, ranked in clusters by ascending order of intensity. Pain intensity was assessed with the 0-10 points Numeric Rating Scale. Each colour relates to the type and number of segments involved in the surgical procedure (decompressive: single level; arthrodesis [ATD]: single or multiple levels).

In the baseline context (eyes open), the analysis of the CoP displacement with different measures of variability for the control and surgery groups (Figure 2) revealed that: a) patients undergoing spine surgery significantly increase their total area of oscillation, and particularly its amplitude in the AP direction; b) the structure of that variability has less complexity and more regularity compared to control participants (significantly lower entropy values). Differences between groups are not significant in the ML direction, and the mean velocity of displacement does not show the ability to discriminate participants (in both directions).

Figure 2: Differences between control (CG) and surgery (SG) groups in terms of the variability metrics used to quantify CoP displacement during the 180 seconds baseline condition (eyes open) trial. SpEn: sample entropy; Range: amplitude of oscillation $(\mathrm{cm})$; Veloc: mean velocity of displacement $(\mathrm{cm} / \mathrm{s})$; Area: the $95 \%$ confidence ellipse area of total displacement $\left(\mathrm{cm}^{2}\right)$; AP: anteroposterior direction; ML: mediolateral direction. The red asterisk indicates statistically significant differences between means ( $\mathrm{p}$-value $<0.05$ ). 
Furthermore, comparing averages from decompression and fusion surgery groups (Figure 3), it turns out that patients submitted to fusion significantly increase their CoP displacement, and have lower values of entropy. However, those features are only significant for the range in the AP direction and the sample entropy in ML direction, against control values, but not the case if compared to decompressive surgery.

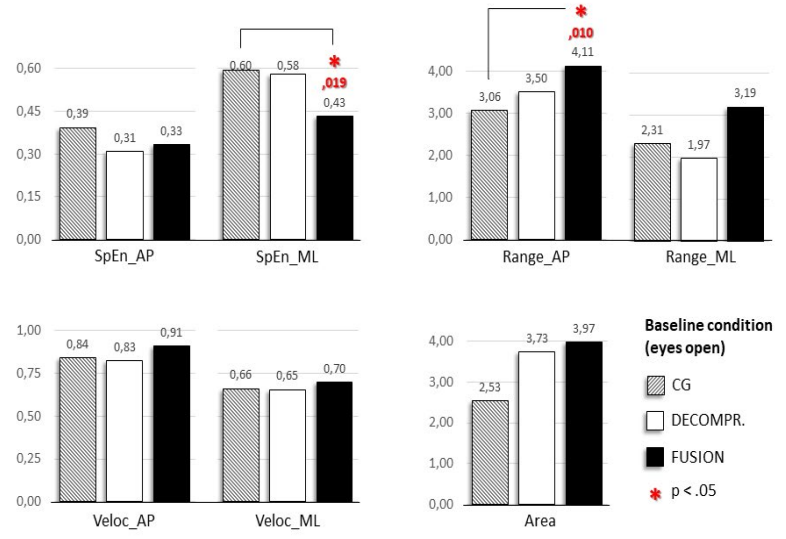

Figure 3: Differences between control, decompression, and fusion surgery groups concerning CoP displacement during baseline condition (eyes open) trial. SpEn: sample entropy; Range: amplitude of oscillation (cm); Veloc: mean velocity of displacement (cm/s); Area: the 95\% confidence ellipse area of total displacement $\left(\mathrm{cm}^{2}\right)$; AP: anteroposterior direction; ML: mediolateral direction. The red asterisk indicates statistically significant differences between means ( $\mathrm{p}$-value $<0.05$ ).

Moreover, the investigation of the level of pain (Figure 4) showed that the report of back or leg pain of level 5 or higher is only significant in provoking a larger area of CoP oscillation, comparing to the control value.
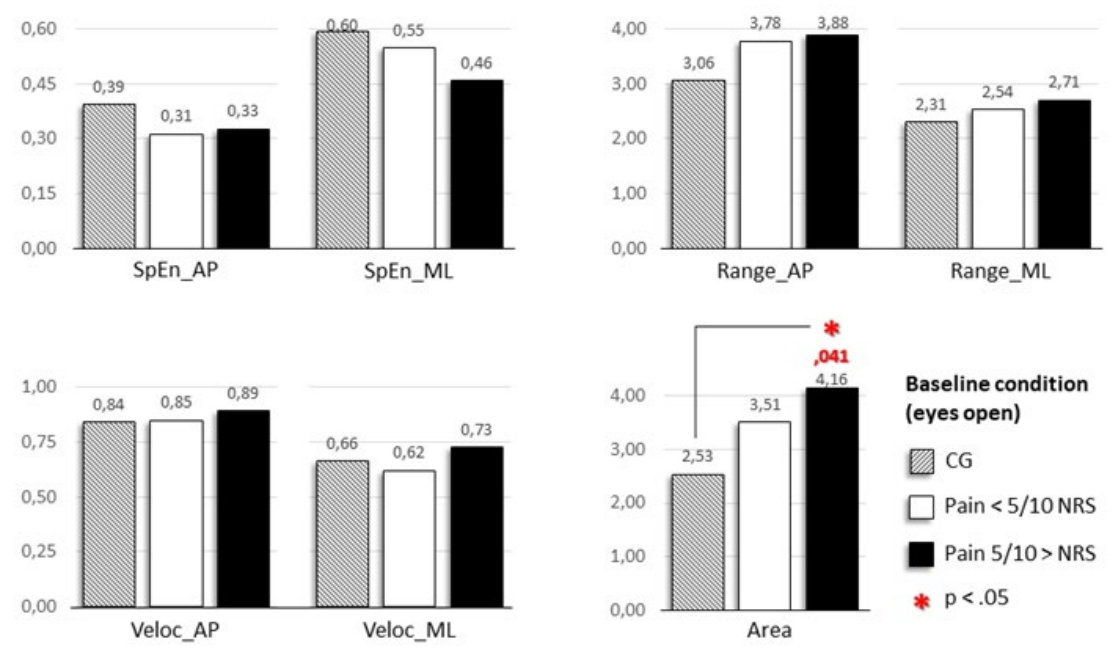

Figure 4: Effects of pain levels on CoP displacement during baseline condition (eyes open) trial. Participants submitted to surgery were ranked in two sub-groups according to their back or leg pain reports: lower than 5; 5 or higher. The 0-10 point Numerical Pain Rating Scale (NRS) was used. SpEn: sample entropy; Range: amplitude of oscillation (cm); Veloc: mean velocity of displacement (cm/s); Area: the 95\% confidence ellipse area of total displacement $\left(\mathrm{cm}^{2}\right)$; AP: anteroposterior direction; ML: mediolateral direction. The red asterisk indicates statistically significant differences between means ( $\mathrm{p}$-value $<0.05$ ). 
Finally, the manifestation of radiculopathy is noted in the significant increase in the amplitude of CoP oscillation in the
AP direction, against control values (Figure 5).
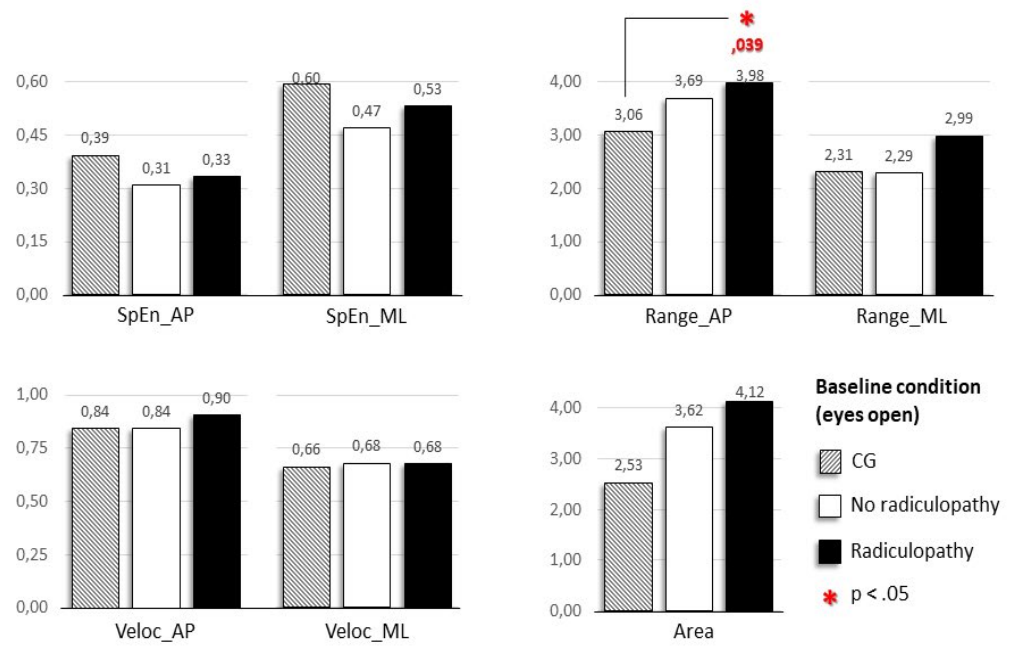

Figure 5: Effects of radiculopathy on CoP displacement during baseline condition (eyes open) trial. Participants submitted to surgery were ranked in two sub-groups according to the presence or not of signs and symptoms of radiculopathy at discharge day (referred leg pain, motor impairment, or disrupted sensation affecting the lower limbs due to lumbosacral nerve root compromise). SpEn: sample entropy; Range: amplitude of oscillation (cm); Veloc: mean velocity of displacement (cm/s); Area: the 95\% confidence ellipse area of total displacement $\left(\mathrm{cm}^{2}\right)$; AP: anteroposterior direction; ML: mediolateral direction. The red asterisk indicates statistically significant differences between means ( $p$-value <0.05).

Analyzing the main effects of the different experimental conditions on the CoP displacement while standing on the platform for the control and surgery groups (Figure 6), it was notable that:

1. Patients exhibited the most significant differences comparing to control participants when they were with eyes closed (EC), on a high-density foam (HD), or holding a small box (BX), displaying a larger area of oscillation, a larger amplitude in both directions (AP/ML); and a faster velocity in the ML direction;

2. The conditions mentioned above also produced significant differences for the sample entropy, but in the opposite way (i.e., patients values lower than controls values);

3. Keeping both feet close together on the platform (FT) induced wider oscillations, but only the range in the ML direction had a significant variance;

4. Performing a dual-task did not have a significant impact, except for the range in the AP direction and the total area;

5. Standing on low-density foam (LD) was the condition with the greatest difference compared to the baseline condition (EO) in both groups, but the disparity was only significant for the entropy and range in the AP direction and the velocity in the ML direction.

Taking into account that the lower the p-value, the stronger the evidence to reject the no-difference hypothesis, a closer look at the $\mathrm{p}$-values resulting from the statistical tests comparing control participants with patients (Table 4 / red asterisks) suggests the relative potential of each condition on the platform to expose significant differences.

The probability values accumulated through all the variability metrics show that the baseline condition had less statistically significant results than the conditions with eyes closed, being on high-density foam, or holding a box, 
which exhibited the stronger significance (Table 4 / yellow rows). Another interesting remark is the absence, in all seven conditions, of significant differences between groups detectable by the mean velocity variable in the AP direction.

\begin{tabular}{|c|c|c|c|c|c|c|c|}
\hline & \multicolumn{2}{|c|}{ Sp. Entropy } & \multicolumn{2}{c|}{ Range } & \multicolumn{2}{c|}{ Velocity } & Area \\
\hline & AP & ML & AP & ML & AP & ML & \\
\hline EO & $*$ & & $*$ & & & & $*$ \\
\hline EC & $* *$ & & $* *$ & $* *$ & & $* *$ & $* *$ \\
\hline FT & & & & $*$ & & & \\
\hline DT & & & $*$ & & & & $*$ \\
\hline HD & $* *$ & $*$ & $*$ & $* *$ & & $* *$ & $*$ \\
\hline LD & $* *$ & & $*$ & & & $*$ & $*$ \\
\hline BX & & $* *$ & $*$ & $* *$ & & $*$ & $* *$ \\
\hline
\end{tabular}

Table 4: P-values from statistical testing comparing mean CoP displacements between control and surgery groups in different stance conditions (EO - eyes open; EC - eyes closed; FT - feet close together; DT - performing a dual-task; HD - on a high-density foam; LD - on a low-density foam; BX - holding a small box), using nonlinear (sample entropy) and linear (range, velocity, and area) measures of variability. AP/ML: anteroposterior and mediolateral directions.

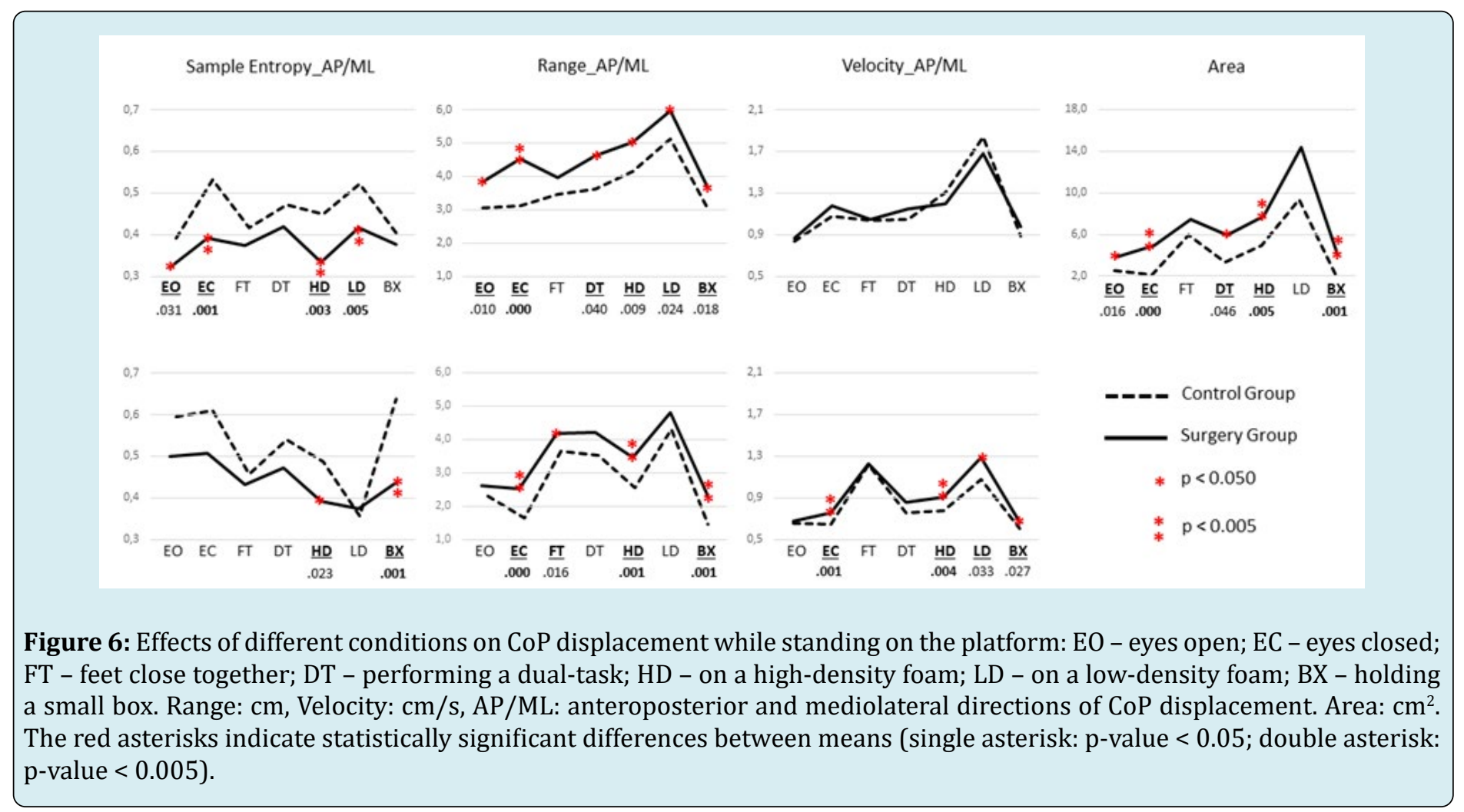

\section{Discussion}

This clinical trial aimed to describe the postural control features of patients who just underwent spine surgery. For that, body sway while standing on a force platform was recorded on the day of hospital discharge after spinal surgery and compared with control values. The same trial was repeated under different stance conditions to clarify which one revealed the best potential to discriminate patients from control participants. This analysis confirmed the interaction of different strategies to overcome the impact of surgery on postural control due to pain or residual radiculopathy and 
the mechanical constraints imposed by the type of surgical technique.

Combining linear and nonlinear tools to provide a comprehensive interpretation of the functionality of postural sway retrieved from stabilometric trials was an innovative approach to explore objective spine surgery outcomes in an early phase. To our knowledge, only Li and colleagues used nonlinear measures but in a two-year follow-up after spine surgery for correction of adolescent idiopathic scoliosis [51]. Other studies had already analysed the variability and complexity of postural patterns in spinal deformities and low back pain [58-61].

Nonlinear dynamics is an alternative or complementary view to the traditional quantitative use of data based on the average values of CoP displacement by appraising the structured temporal organisation of motor behaviour, in the form of how a body sways to keep balanced along the time-series. The focus is changed from the stated level of motor control to the type of motor strategy adopted to solve postural and dynamic task demands depending on the actual constraints of the system [62].

Evidence about the interaction between back pain or spinal deformities and postural sway is not consensual, although the majority of systematic reviews suggest an overall tendency to an increased oscillation $[63,64]$, and a loss of complexity and higher regularity of postural patterns [59]. In this study, during the baseline task (eyes open, feet apart, on a firm surface, performing a single task), patients are distinguished from controls in that they sway significantly more in terms of total area and especially in the AP direction. The classical biomechanical assumption is that they have worse control of their posture and balance [65], which is expected considering the acute iatrogenic effect of surgery. It may be argued that the alterations were due to orthostatic hypotension. However, all the patients had a controlled clinical status with an adjusted hemodynamic response without vagal reaction that could interfere with balance, being part of the criteria to justify the cessation of hospital care and discharge.

Sample entropy values from our patients were the opposite of the other (linear) variables, being lower than control values, again more significantly in the AP direction. This data is interpreted as a postural pattern markedly regular and less complex, typically found in systems with poor flexibility and limited reply to perturbations. The highest values of entropy are associated with excessive and random irregularity, indicating a notably unstable system likely to relate with a strongly impaired postural control. Finally, a system without restrictions and moderately irregular though structured postural behaviour, denoting more variability and better adaptation to perturbations, would display entropy values in between [66].

Going deeper into the isolated effect of different parameters (the type of surgery, level of pain and presence of radiculopathy), splitting the surgery group into the corresponding pairs under scrutiny, did not reveal a substantial difference, apart from a slight increase in the sway magnitude of fusion patients; those with pain level 5 or more; or in the presence of radicular impairment. Nevertheless, no statistical significance distinguished groups of patients, except in an isolated confront with control values.

\section{Effect of the Type of Surgery}

Intervertebral fusion is expected to increase trunk stiffness, apart of correcting or at least altering spinal alignment, having in mind the reduction of range of movements in the degrees of freedom available, proportional to the number of fused levels. However, sample entropy values from the whole sub-group of fusion patients differ only from decompressive surgery in the ML direction and only significantly compared to control participants. Apparently, the restrictions due to arthrodesis are more critical in the frontal plane, reducing the options to solve balance issues in the ML direction. Accordingly, the linear quantification of CoP displacement revealed higher values of amplitude and total area than the control reference, but the most substantial difference between decompression and fusion was noticed only in the ML direction.

Thoracolumbar fusion (T4-L3 / 11 intervertebral levels) to correct adolescent idiopathic scoliosis (AIS) represents the highest impact of surgery on spine mobility. The exclusive data from the three younger patients who submit this technique should corroborate the previous assumptions. Likewise, values of entropy, amplitude, and the total area of CoP displacement detaches significantly from decompressive surgery; yet, that disparity is observed only in the AP direction. This contradiction may be explained by the discrepancy between the extensions of lumbar/lumbosacral and thoracolumbar fusions, with different impacts on trunk and pelvis mobility. Nevertheless, the small sample represented by the late sub-group in this study precludes any further generalisation.

$\mathrm{Li}$ and colleagues observed comparable CoP sway complexity in AIS patients versus healthy controls in a twoyear follow-up after surgery [51], suggesting that mechanical restrictions due to arthrodesis may not explain the results entirely in an early stage after surgery. In contrast, Piatek and colleagues [67] found lower sample entropy values by 


\section{Journal of Orthopedics \& Bone Disorders}

AIS patients wearing trunk braces. On the other way, the correction of spinal curvatures may solve the reduction of complexity due to previous focus on the control of the trunk shift, as pointed by Gruber and colleagues [59]. The repositioning of the sagittal balance seems to have a crucial role in the outcomes $[48,68]$. Similarly, a positive impact on the vestibular system after spinal fusion was described by Delpierre and colleagues [69], resulting from the reduction of trunk displacement and, therefore, of the head, with improved balance as a consequence. Nonetheless, the iatrogenic muscle damage caused by spinal fusion [45] may impair their activation, strength, and flexibility, besides being painful in the early stage, imposing an additional impact on the mentioned biomechanical constraints. In any case, these consequences are also present in decompression surgery, so that may explain the absence of a significant difference between the types of surgery.

\section{Effect of the Level of Pain}

Entropy values from patients were lower than control participants, regardless of the referred pain level. Although not significantly, most complaining patients emerged with the largest difference having the lowest values in the ML direction. When confronted side by side, entropy graphics reporting differences between types of surgery and levels of pain are quite similar, which seems to be associated with the predominance of fusion surgery (14 [70\%] in 20 subjects) among the most complaining.

Both clinical practise and research provide indications that subjects with lumbopelvic dysfunction associated with pain present less variability concerning postural control strategies [70,71]. Alternative forms of trunk mobility control in the presence of pain or injury are described, relying on an over activation of the strong superficial muscles of trunk, pelvis, and hip, as a solution to increase protective stiffness [72]. This pattern of co-contraction between antagonists (trunk flexors/extensors) and bilateral muscles (hip abductors) provides a static, though rigid stability, that profound muscles can no longer ensure due to inhibition, reducing, or delaying their activation [18]. It is plausible that this configuration is present on the most complaining patients within the fusion group, evoking a more regular pattern of motor behaviour in the frontal plane.

Somehow, the magnitude of pain reports does not follow a proportional distribution according to the complexity of surgery procedures. Cognitive and psychosocial factors are known to interfere with the perception of discomfort, depending on previous experience with back pain and injury, beliefs about the potential threatening from exposition to functional demands, or the availability of sensorial feedback to improve control [15].

\section{Effect of The Radiculopathy}

The presence of residual radiculopathy after surgery with proprioceptive and motor impairment may interfere with the available sensorial input $[20,73]$, reducing the effectiveness of reactive postural adjustments, and give rise to insecurity and defence, even in the absence of pain $[22,74]$. The anticipation of pain is known to evoke protective postural strategies that stiff the spine [75]. Furthermore, chronic back pain, a common feature of spine surgery patients, is closely related to fear-avoidance beliefs and maladaptive cognitive processes associated to catastrophisation and kinesiophobia, which are predictive of marked changes in motor behaviour [76].

Overall, the neuromuscular system is expected to lose its ability to provide the coordinated dynamic stability needed to optimise postural control [12] and lacks the natural variability for an adjusted reply to postural demands [66]. Nonetheless, Mazaheri and colleagues have not found any competing effect of fear of pain in postural control, and the increased regularity of sway patterns described in the low back pain patients studied was attributable to current pain only [77].

In this study, patients with radiculopathy follow the same tendency to increase their postural sway in terms of amplitude and total area, significantly in the AP direction only when matched to control participants. No significant difference was observed compared to radiculopathy-free patients, though more discrepant in the ML direction, close to what fusion patients had demonstrated. These late results agree with those of Rosker and colleagues [44], pointing significant changes in the mediolateral sway in patients after spinal surgery treating lumbar disk herniation, suggesting that postural balance deficits are side-specific, due to the nature of the pathology. Entropy measures in this sub-group maintained the trend of more regular and less complex oscillations (lower values than control references), again, without significance comparing patients or between them and control participants.

\section{Effect of the Stance Condition}

Mechanic, sensory, and cognitive manipulation were performed, modifying the original stance condition on the platform. The aim was to analyse the effect on CoP displacement and find which conditions are more sensitive to the differences between participants, providing a better insight into the motor strategies used by spine surgery patients, and help to clarify the underlying mechanisms that drive balance control impairment and guide rehabilitation approaches. 
Therefore, the participants were engaged to perform other tasks, standing on the platform:

- Eyes closed - Previous studies suggest that removing visual input would enhance the influence of proprioceptive and vestibular impairment on postural control, revealing a greater magnitude of postural sway [40];

- Dual-task - An additional cognitive task demands shared attention that subtracts resources needed to optimise postural performance and balancing maintenance. Body sway regularity increases as the amount of attention are devoted to posture, depending on the balance demands, or reduces by performing a dual-task [78,79]. Patients executed a backward counting task;

- Feet together - A narrower base of support is a more challenging balance task that may be used to evoke the late effects [80];

- Foam - In the same way, the stability of the surface can be manipulated using foam with different densities to explore the dependency on the proprioceptive system [81]. Participants were invited to stand on high (55 kg/ $\left.\mathrm{m}^{3}\right)$ and low-density foam $\left(35 \mathrm{~kg} / \mathrm{m}^{3}\right)$ in two different moments;

- Holding a box - Considering the significant influence that psycho-emotional aspects have on motor behaviour $[82,83]$, the postural response to a task that could invoke emotional states of fear and insecurity, associated with behaviours of avoidance or immobility were explored. In our case, holding a box should represent a potentially dangerous task after spine surgery, although its safe weight of only $6 \%$ of the participant's body weight $[84,85]$.

Analysis of the results confirmed that the baseline condition is not the best option to have the most defined picture about the impact of a surgery over the sensorimotor system and the strategies to integrate available sensory inputs. As expected, the eyes-closed condition provided statistically significant information in almost all the variables.

A noteworthy result was the fact that the use of the highest density foam was comparable in statistical strength to the eyes closed, with the same potential to expose differences between patients by uncovering proprioceptive deficits not compensated by the visual system. Interestingly, although the low-density foam was the most challenging balance condition, the results demonstrated a lower sensitivity to discriminate participants, and the AP values of sample entropy revealed the most significant difference. Probably, due to the large amount of attention needed to its performance, both patients and controls were forced to use the same safety strategies in the frontal plane, but patients used a much higher regularity in the sagittal sway, even at the expense of significative larger amplitude and loss of automatism [78].

Although Mazaheri and colleagues' [77] study showed that there was no competing effect of fear of pain, the concurrent task of holding a box while standing for 3 minutes may have demanded an attentional investment particularly high, according to the perceived level of potential risk [86]. This task provoked as well an augmented sway and a postural strategy of more regularity, but this time in the ML direction, a pattern found in higher demanding conditions [87] or the presence of pain [88]. Particularly in the case of chronic pain, the association with maladjusted catastrophising and kinesiophobia behaviours is quite strong [89], with a significant influence on posture and movement patterns [15]. The manipulation of these parameters may give clues about the degree of influence of the affective component on the postural control.

Narrowing the base of support did not bring any particular information except the close relationship with a significant increase in the ML sway, which is tuned with the direction of narrowing. Likewise, the cognitive task of backward counting was only significant to reveal an increase in the area and AP body swing, perhaps not sufficiently demanding to produce further effects.

The absence of a pre-operative assessment and a control group of patients are the main limitations of the present study. Equally interesting would be a follow-up of at least 3 months and 1 year. In addition to pain intensity, other subjective data should have been taken, like measures of functionality, health-related quality of life, and psychological distress.

Despite the high heterogeneity of pre-operative spine conditions and types of surgery procedures being an advantage due to the broader scope of the study, and an innovative aspect in a clinical trial on postural control after spine surgery, the small size of each subgroup prevented the possibility of studying the interaction effect of the proposed parameters (the type of surgery, level of pain, radiculopathy). Participants were included in a wide age range that enriched the study again; the almost complete match between control and surgery groups solved any age-related bias.

Another limitation is the possibility of induced bias by fatigue and discomfort due to the prolonged time needed to fulfil all the seven tasks on the platform for three minutes each. Future studies should keep the duration of each trial to use nonlinear measures that benefit from longer time-series, but restrict the stance conditions to the most relevant, at least eyes open, eyes closed, and the high-density foam.

To our knowledge, this study was the first to apply a 
combined approach using linear and nonlinear measures of variability to assess postural control at hospital discharge after spine surgery. Future studies should dedicate to explore both the magnitude and the structure of posture and movement variability, emphasising the use of a broader set of nonlinear tools.

A comprehensive understanding of the underlying motor strategies adopted by patients with spine conditions in response to surgery at an early stage allows us to better targeting therapeutic interventions to improve recovery and overcome the remaining habitual discrepancy in the longterm. Implementing early treatment after spine surgery should be recommended, based on strategies to facilitate the acquisition of correct motor patterns, aiming to regain postural control and reinforce optimal movement variability to achieve functional autonomy and prevent recurrence and chronicity.

\section{Conclusion}

At hospital discharge, patients having surgery for spine conditions show a significant increase in the magnitude of body sway and a postural pattern markedly regular with low complexity.

These features are evidenced by higher values of total area and AP range of CoP displacement, and by lower values of AP sample entropy, measured during a 180s standard stabilometric trial and compared with an age and gender match of healthy volunteers.

Stabilometric trials performed with eyes closed, on highdensity foam, or holding a box, demonstrate superior ability to discriminate patients from controls.

In this study, fusion surgery, referred back or leg pain equal or higher than 5/10 NRS, and signs or symptoms of residual radiculopathy were only marginally responsible for the postural patterns adopted. Further studies using nonlinear measures after spine surgery are needed to clarify the underlying motor behaviour. Early intervention is recommended to correct postural impairments and facilitate optimal movement variability.

\section{References}

1. James SL, Abate D, Abate KH, Abay SM, Abbafati C, et al. (2018) Global, regional, and national incidence, prevalence, and years lived with disability for 354 diseases and injuries for 195 countries and territories, 1990-2017: a systematic analysis for the Global Burden of Disease Study 2017. The Lancet 392(10159): 17891858.
2. Foster NE, Anema JR, Cherkin D, Chou R, Cohen SP, et al. (2018) Prevention and treatment of low back pain: evidence, challenges, and promising directions. The Lancet 391(10137): 2368-2383.

3. Chou R, Baisden J, Carragee EJ, Resnick DK, Shaffer WO, et al. (2009) Surgery for low back pain: a review of the evidence for an American Pain Society Clinical Practice Guideline. Spine 34(10):1094-1109.

4. Deyo RA, Mirza SK (2016) Herniated lumbar intervertebral disk. New England Journal of Medicine 374(18): 1763-1772.

5. NICE_UK (2016) Low Back Pain and Sciatica in Over 16s: Assessment and Management: National Institute for Health and Care Excellence (UK).

6. Chou R, Loeser JD, Owens DK, Rosenquist RW, Atlas SJ, et al. (2009) Interventional Therapies, Surgery, and Interdisciplinary Rehabilitation for Low Back Pain: An Evidence-Based Clinical Practice Guideline From the American Pain Society. Spine 34(10): 1066-1077.

7. Tousignant-Laflamme Y, Christopher S, Clewley D, Ledbetter L, Cook CJ, et al. (2017) Does shared decision making results in better health related outcomes for individuals with painful musculoskeletal disorders? A systematic review. The Journal of Manual \& Manipulative Therapy 25(3): 144-150.

8. Relvas H, Fernandes O, Oliveira R (2018) Outcome Measures in Spine Surgery: How Far Can we Go Building Evidence? J Ortho Bone Disord 2(3): 000161.

9. Chiarotto A, Boers M, Deyo RA, Buchbinder R, Corbin TP, et al. (2018) Core outcome measurement instruments for clinical trials in nonspecific low back pain. Pain 159(3): 481-495.

10. Waddell G, Somerville D, Henderson I, Newton M (1992) Objective clinical evaluation of physical impairment in chronic low back pain. Spine 17(6): 617-628.

11. Papi E, Bull AMJ, McGregor AH (2018) Is there evidence to use kinematic/kinetic measures clinically in low back pain patients? A systematic review. Clinical biomechanics 55: 53-64.

12. Reeves NP, Cholewicki J, Pearcy M, Parnianpour M (2013) How can models of motor control be useful for understanding low back pain? In: Hodges PW, Cholewicki J, van_Dieen JH, editors. Spinal Control: Churchill Livingstone, pp: 187-193.

13. Xu Y, Choi J, Reeves NP, Cholewicki J (2010) Optimal control of the spine system. Journal of biomechanical 
engineering 132: 51004.

14. Brumagne S, Janssens L, Knapen S, Claeys K, SuudenJohanson E (2008) Persons with recurrent low back pain exhibit a rigid postural control strategy. European spine journal : official publication of the European Spine Society, the European Spinal Deformity Society, and the European Section of the Cervical Spine Research Society 17(9): 1177-1184.

15. Hodges PW, Tucker K (2011) Moving differently in pain: a new theory to explain the adaptation to pain. Pain 152(3): S90-S98.

16. Hildebrandt M, Fankhauser G, Meichtry A, Luomajoki $H$ (2017) Correlation between lumbar dysfunction and fat infiltration in lumbar multifidus muscles in patients with low back pain. BMC musculoskeletal disorders 18: 12.

17. Hodges PW, James G, Blomster L, Hall L, Schmid AB, et al. (2014) Can proinflammatory cytokine gene expression explain multifidus muscle fiber changes after an intervertebral disc lesion? Spine 39(13): 1010-1017.

18. Hodges PW, Richardson CA (1998) Delayed postural contraction of transversus abdominis in low back pain associated with movement of the lower limb. Journal of spinal disorders 11(1): 46-56.

19. Moseley GL, Hodges PW (2005) Are the changes in postural control associated with low back pain caused by pain interference? The Clinical journal of pain 21(4): 323-329.

20. Leinonen V, Kankaanpaa M, Luukkonen M, Kansanen M, Hanninen 0, et al. (2003) Lumbar paraspinal muscle function, perception of lumbar position, and postural control in disc herniation-related back pain. Spine 28(8): 842-848.

21. Morag E, Hurwitz DE, Andriacchi TP, Hickey M, Andersson GBJ (2000) Abnormalities in Muscle Function During Gait in Relation to the Level of Lumbar Disc Herniation. Spine 25(7): 829-833.

22. Sipko T, Chantsoulis M, Kuczyński M (2010) Postural control in patients with lumbar disc herniation in the early postoperative period. European spine journal : official publication of the European Spine Society, the European Spinal Deformity Society, and the European Section of the Cervical Spine Research Society 19(3): 409-414.

23. Sipko T, Chantsoulis-Supinska M, Zmuda M, Zwolinski J (2008) Postural balance in the early postoperative period in patients with intervertebral disk disease following surgery. Ortopedia, traumatologia, rehabilitacja 10(3): 226-237.

24. Sawatzky B, Tredwell S, Sanderson D (1997) Postural control and trunk imbalance following Cotrel-Dubousset instrumentation for adolescent idiopathic scoliosis. Gait \& posture 5(2): 116-119.

25. Gage WH, Winter DA, Frank JS, Adkin AL. (2004) Kinematic and kinetic validity of the inverted pendulum model in quiet standing. Gait \& posture 19(2): 124-132.

26. Winter DA, Prince F, Frank JS, Powell C, Zabjek KF (1996) Unified theory regarding A/P and M/L balance in quiet stance. Journal of neurophysiology 75(6): 2334-2343.

27. Valles KD, Long JT, Riedel SA, Graf A, Krzak J, Hassani $S$, et al. (2009) Using a bi-planar postural stability model to assess children with scoliosis. Conference proceedings : Annual International Conference of the IEEE Engineering in Medicine and Biology Society IEEE Engineering in Medicine and Biology Society Conference pp: 7010-7013.

28. Prieto TE, Myklebust JB, Hoffmann RG, Lovett EG, Myklebust BM (1996) Measures of postural steadiness: differences between healthy young and elderly adults. IEEE Trans Biomed Eng 43(9): 956-966.

29. Latash ML, Scholz JP, Schoner G (2002) Motor control strategies revealed in the structure of motor variability. Exerc Sport Sci Rev 30(1): 26-31.

30. van Emmerik RE, van Wegen EE (2002) On the functional aspects of variability in postural control. Exerc Sport Sci Rev 30(4): 177-183.

31. Riley MA, Turvey MT (2002) Variability of determinism in motor behavior. J Mot Behav 34(2): 99-125.

32. Harbourne RT, Stergiou N (2009) Movement variability and the use of nonlinear tools: principles to guide physical therapist practice. Physical therapy 89(3): 267282.

33. Stergiou N, Decker LM (2011) Human movement variability, nonlinear dynamics, and pathology: is there a connection? Human movement science 30(5): 869-888.

34. Stergiou N (2016) Nonlinear Analysis for Human Movement Variability: CRC Press

35. Nies N, Sinnott PL (1991) Variations in balance and body sway in middle-aged adults. Subjects with healthy backs compared with subjects with low-back dysfunction. Spine 16(3): 325-330. 
36. Mazaheri M, Coenen $P$, Parnianpour M, Kiers H, van Dieen JH (2013) Low back pain and postural sway during quiet standing with and without sensory manipulation: a systematic review. Gait \& posture 37(1): 12-22.

37. Ruhe A, Fejer R, Walker B (2011) Center of pressure excursion as a measure of balance performance in patients with non-specific low back pain compared to healthy controls: a systematic review of the literature. European spine journal : official publication of the European Spine Society, the European Spinal Deformity Society, and the European Section of the Cervical Spine Research Society 20(3): 358-368.

38. Berenshteyn Y, Gibson K, Hackett GC, Trem AB, Wilhelm $M$ (2019) Is standing balance altered in individuals with chronic low back pain? A systematic review 41(13): 1514-1523.

39. Koch C, Hänsel F (2019) Non-specific Low Back Pain and Postural Control During Quiet Standing-A Systematic Review. Frontiers in psychology 10: 586.

40. Yen TC, Toosizadeh N, Howe C, Dohm M, Mohler J, et al. (2016) Postural Balance Parameters as Objective Surgical Assessments in Low Back Disorders: A Systematic Review. Journal Applied Biomechanics 32(3): 316-323.

41. Scoppa F, Capra R, Gallamini M, Shiffer R (2013) Clinical stabilometry standardisation: basic definitions-acquisition interval--sampling frequency. Gait Posture 37(2): 290-292.

42. Przysada G, Guzik A, Rosak-Matuszewska I, Druzbicki M, Wolan-Nieroda A, et al. (2018) Posture control in patients with herniated nucleus pulposus in cervical and lumbosacral spine subjected to operative treatment. Journal Back Musculoskeletal Rehabilitation 31(5): 795802 .

43. de Abreu DC, Gomes MM, de Santiago HA, Herrero CF, Porto MA, et al. (2012) What is the influence of surgical treatment of adolescent idiopathic scoliosis on postural control?. Gait Posture 36(3): 586-590.

44. Rosker ZM, Rosker J, Sarabon N (2020) Impairments of Postural Balance in Surgically Treated Lumbar Disc Herniation Patients. J Appl Biomech 1-7.

45. Pao JL, Yang RS, Hsiao CH, Hsu WL (2014) Trunk Control Ability after Minimally Invasive Lumbar Fusion Surgery during the Early Postoperative Phase. J Phys Ther Sci 26(8): 1165-1171.

46. de Santiago HA, Reis JG, Gomes MM, da Silva Herrero CF, Defino HL, et al. (2013) The influence of vision and support base on balance during quiet standing in patients with adolescent idiopathic scoliosis before and after posterior spinal fusion. Spine Journal 13(11): 1470-1476.

47. Wong WJ, Lai DM, Wang SF, Wang JL, Hsu WL (2019) Changes of balance control in individuals with lumbar degenerative spine disease after lumbar surgery: a longitudinal study. Spine Journal 19(7): 1210-1220.

48. Gottipati P, Stine R, Ganju A, Fatone S (2018) The effect of positive sagittal spine balance and reconstruction surgery on standing balance. Gait Posture 62: 227-234.

49. Yagi M, Ohne H, Kaneko S, Machida M, Yato Y, et al. (2018) Does corrective spine surgery improve the standing balance in patients with adult spinal deformity?. Spine Journal 18(1): 36-43.

50. Schimmel JJ, Groen BE, Weerdesteyn V, de Kleuver M (2015) Adolescent idiopathic scoliosis and spinal fusion do not substantially impact on postural balance. Scoliosis 10: 18 .

51. Li Y, Kakar RS, Fu Y-C, Walker M, Brown CN, et al. (2019) Postural control of individuals with spinal fusion for adolescent idiopathic scoliosis. Clinical Biomechanics 61: 46-51.

52. Valles KB, Long J, Riedel S, Graf A, Krzak J, et al. (2010) Analysis of postural stability following posterior spinal fusion in adolescents with idiopathic scoliosis. Studies Health Technology Informatics 158: 127-31.

53. Bouche K, Stevens V, Cambier D, Caemaert J, Danneels $\mathrm{L}$ (2006) Comparison of postural control in unilateral stance between healthy controls and lumbar discectomy patients with and without pain. European spine journal 15(4): 423-432.

54. Farrar JT, Young Jr JP, LaMoreaux L, Werth JL, Poole RM (2001) Clinical importance of changes in chronic pain intensity measured on an 11-point numerical pain rating scale. Pain 94(2): 149-158.

55. Karcioglu O, Topacoglu H, Dikme O, Dikme 0 (2018) A systematic review of the pain scales in adults: Which to use?. Am J Emerg Med 36(4): 707-714.

56. Markman JD, Czerniecka-Foxx K, Khalsa PS, Hayek SM, Asher AL, et al. (2020) AAPT Diagnostic Criteria for Chronic Low Back Pain. J Pain.

57. Richman JS, Moorman JR (2000) Physiological timeseries analysis using approximate entropy and sample entropy. American Journal of Physiology-Heart and Circulatory Physiology 278(6): 2039-2049. 
58. Piątek E, Kuczyński M, Ostrowska B (2020) The effects of active self-correction on postural control in girls with adolescent idiopathic scoliosis: The role of an additional mental task. International Journal Environmental Research Public Health 17(5): 1640.

59. Gruber AH, Busa MA, Gorton Iii GE, Van Emmerik RE, Masso PD, et al. (2011) Time-to-contact and multiscale entropy identify differences in postural control in adolescent idiopathic scoliosis. Gait \& posture 34(1): 1318.

60. Fewster KM, Gallagher KM, Howarth SH, Callaghan JP (2020) Low back pain development differentially influences centre of pressure regularity following prolonged standing. Gait Posture 78: e1-e6.

61. Shahvarpour A, Preuss R, Larivière C (2019) The effect of extensible and non-extensible lumbar belts on trunk postural balance in subjects with low back pain and healthy controls. Gait Posture 72: 211-216.

62. Decker LM, Moraiti C, Stergiou N, Georgoulis AD (2011) New insights into anterior cruciate ligament deficiency and reconstruction through the assessment of knee kinematic variability in terms of nonlinear dynamics. Knee Surg Sports Traumatol Arthrosc 19: 1620-1633.

63. Kiers H, van Dieën JH, Brumagne S, Vanhees L (2015) Postural sway and integration of proprioceptive signals in subjects with LBP. Human Movement Science 39: 109120.

64. Ruhe A, Fejer R, Walker B (2011) Is there a relationship between pain intensity and postural sway in patients with non-specific low back pain? BMC musculoskeletal disorders 12: 162.

65. Cavanaugh JT, Guskiewicz KM, Stergiou N (2005) A nonlinear dynamic approach for evaluating postural control: new directions for the management of sportrelated cerebral concussion. Sports Med 35: 935-950.

66. Stergiou N, Harbourne R, Cavanaugh J (2006) Optimal movement variability: a new theoretical perspective for neurologic physical therapy. J Neurol Phys Ther 30(3): 120-129.

67. Piątek E, Kuczyński M, Ostrowska B (2019) Postural control in girls with adolescent idiopathic scoliosis while wearing a Chêneau brace or performing active selfcorrection: a pilot study. Peer J 7: e7513.

68. Yagi M, Kaneko S, Yato Y, Asazuma T (2017) Standing Balance and Compensatory Mechanisms in Patients With Adult Spinal Deformity. Spine 42(10): E584-E591.
69. Delpierre Y, Vernet P, Colin D (2020) Does spinal fusion influence vestibular information in scoliosis patients? Unstable equilibrium analysis. Journal Orthopaedics 20: 280-285.

70. Brumagne S, Janssens L, Janssens E, Goddyn L (2008) Altered postural control in anticipation of postural instability in persons with recurrent low back pain. Gait Posture 28: 6576-62.

71. Hodges PW, Cholewicki J (2007) Functional control of the spine. In: Vleeming A, Mooney V, Stoeckart R, (Eds.), Movement, Stability \& Lumbopelvic Pain, $2^{\text {nd }}$ (Edn.), Churchill Livingstone, Edinburgh, pp: 489-512.

72. McGill SM, Grenier S, Kavcic N, Cholewicki J (2003) Coordination of muscle activity to assure stability of the lumbar spine. Journal Electromyography Kinesiology 13(4): 353-539.

73. Brumagne S, Cordo P, Verschueren S (2004) Proprioceptive weighting changes in persons with low back pain and elderly persons during upright standing. Neuroscience Letters 366: 63-66.

74. MacDonald D, Moseley GL, Hodges PW (2010) People with recurrent low back pain respond differently to trunk loading despite remission from symptoms. Spine 35(7): 818-824.

75. Moseley GL, Nicholas MK, Hodges PW (2004) Does anticipation of back pain predispose to back trouble? Brain Journal Neurology 127(10): 2339-2347.

76. Haddas R, Lieberman IH, Block A (2018) The Relationship Between Fear-Avoidance and Objective Biomechanical Measures of Function in Patients With Adult Degenerative Scoliosis. Spine 43(9):647-653.

77. Mazaheri M, Heidari E, Mostmand J, Negahban H, van Dieen JH (2014) Competing Effects of Pain and Fear of Pain on Postural Control in Low Back Pain? Spine 39(25): 1518-1523.

78. Roerdink M, Hlavackova P, Vuillerme N (2011) Centerof-pressure regularity as a marker for attentional investment in postural control: A comparison between sitting and standing postures. Human Movement Science 30(2): 203-212.

79. Donker SF, Roerdink M, Greven AJ, Beek PJ (2007) Regularity of center-of-pressure trajectories depends on the amount of attention invested in postural control. Experimental Brain Research 181(1): 1-11.

80. da Silva RA, Vieira ER, Fernandes KBP, Andraus RA, Oliveira MR, et al. (2018) People with chronic low back 
pain have poorer balance than controls in challenging tasks. Disability Rehabilitation 40(11): 1294-1300.

81. Claeys K, Brumagne S, Dankaerts W, Kiers H, Janssens L (2011) Decreased variability in postural control strategies in young people with non-specific low back pain is associated with altered proprioceptive reweighting. European Journal Applied Physiology 111: 115-123.

82. Horslen BC, Carpenter MG (2011) Arousal, valence and their relative effects on postural control. Experimental Brain Research 215(1): 27-34.

83. Archer KR, Seebach CL, Mathis SL, Riley LH, Wegener ST (2014) Early postoperative fear of movement predicts pain, disability, and physical health six months after spinal surgery for degenerative conditions. The Spine Journal 14(5): 759-767.

84. Lu M-L, Waters TR, Krieg E, Werren D (2014) Efficacy of the revised NIOSH lifting equation to predict risk of low-back pain associated with manual lifting: a one-year prospective study. Human Factors 56(1): 73-85.

85. Roberts M, Talbot C, Kay A, Price M, Hill M (2018)
Changes in postural sway and gait characteristics as a consequence of anterior load carriage. Gait Posture 66: 139-145.

86. Stins JF, Michielsen ME, Roerdink M, Beek PJ (2009) Sway regularity reflects attentional involvement in postural control: Effects of expertise, vision and cognition. Gait Posture 30(1): 106-109.

87. Shahbazi Moheb Seraj M, Sarrafzadeh J, Maroufi N, Ebrahimi Takamjani I, Ahmadi A, et al. (2019) Comparison of Postural Balance between Subgroups of Nonspecific Low-back Pain Patients Based on O'Sullivan Classification System and Normal Subjects during Lifting. The Archives Bone Joint Surgery 7(1): 52-60.

88. Stins JF, Roerdink M, Beek PJ (2011) To freeze or not to freeze? Affective and cognitive perturbations have markedly different effects on postural control. Human Movement Science 30(2): 190-202.

89. Rainville J, Smeets RJEM, Bendix T, Tveito TH, Poiraudeau S, et al. (2011) Fear-avoidance beliefs and pain avoidance in low back pain-translating research into clinical practice. The Spine Journal 11(9): 895-903. 